\title{
Discussion of Scattered Shunting Toll Plaza
}

\author{
Yinhe Shen ${ }^{*}$ \\ School of Electrical Engineering, North China Electric Power University, Baoding 071000, China \\ 466738331@qq.com
}

\begin{abstract}
We address the problem of congestion through traffic shunting. Thus, we establish the Traffic Shunting Model to divide the traffic flow. Based on the Traffic Linear Model, we test the throughput of our model. To simplify the calculation of total traffic flow after merging, we define the Traffic Flow Block Model creatively. Under the circumstance of qualitative analysis, our model is indeed able to decrease the traffic flow density through the subsequent simulation, so as to increase the total traffic throughput effectively.
\end{abstract}

Keywords: Traffic Shunting Model, traffic flow.

\section{Scattered Distribution Model}

\subsection{Model Construction}

In this section, we design the scattered distribution model of toll plaza by staggering the road line of two directions. We stagger the toll station of each direction, and increase the number of fan-in part in each direction, which is beneficial to both the increase of number of toll station and the utilization of land.

From the point of construction costs, compared with the normal one, our model can improve the utilization of highway by making the waste land outside the arc edge, thus decrease the cost of land and road construction.

From the aesthetic principle of urban planning, the gradual curve is more aesthetic, and on the other hand, reducing the angle of curve can decrease the accident rate effectively.

The diagram of scattered distribution model can be shown in Fig. 1.

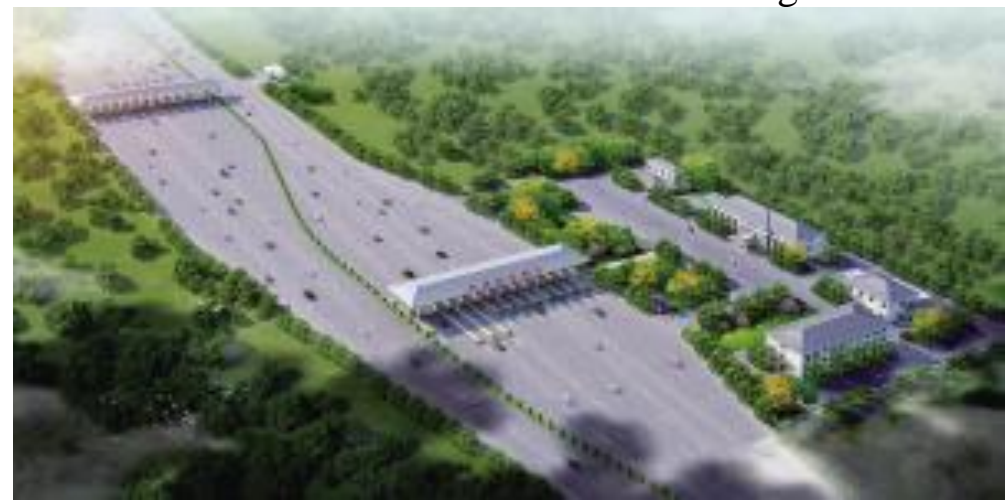

Fig. 1. Scattered distribution model

\subsection{Model Testing on Cost}

We think that the main factors affecting the cost would be the construction cost of toll plaza and tollbooth. The construction cost of each square meters is knowable. And because of the different cost of three kinds of tollbooths, the comprehensive construction cost of tollbooths is a function of the proportion of ETC, MTC and conventional tollbooths. Based on the conclusion of last question, the proportion is stable, hence the comprehensive construction cost of tollbooths is also knowable. Finally, we can get a cost function:

$$
C=p_{1} S+p_{2}
$$

After a simple analysis, the cost function is the function of area. Therefore, to optimize the total cost, we aim to decrease the area to achieve the goal. Based on this idea, we establish the Staggered Model to explore this question. The comparison of traditional model with the Staggered Model can be shown in Fig. 2. 


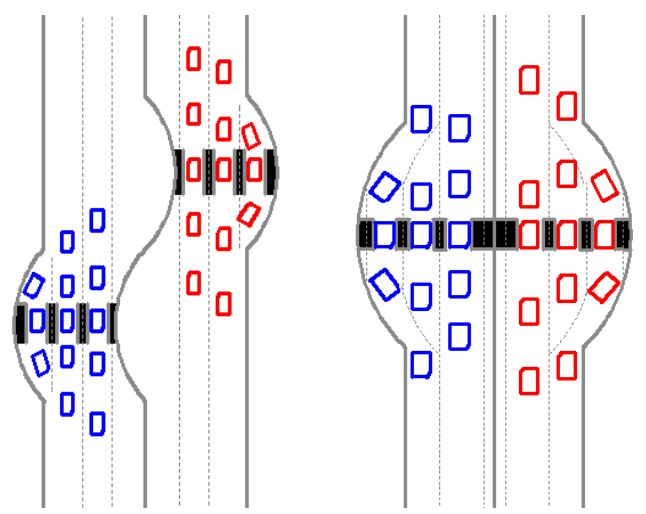

Fig. 2. comparison of traditional model with the Staggered Model

From the comparison, it can be seen that we take advantage of internal space of highway fully by setting the fan in and fan out areas in the interior. And we only set half fan in and fan out regions outside the highway. In this way, we decrease the total needy area of toll plaza.

\section{Traffic-Shutting Model}

\subsection{Model Construction}

Further, we optimize our model by taking the shunting into consideration. As we know, the way of merging in common toll plaza is based on the only one fan-ion part. In the scattered distribution model, we increase the number of fan-in part to twice. In this part, we turn the merging on both sides at the same time to merging at different time, in order to decrease the traffic density in each merging process.

The diagram of Traffic-Shutting model can be shown in Fig. 3.

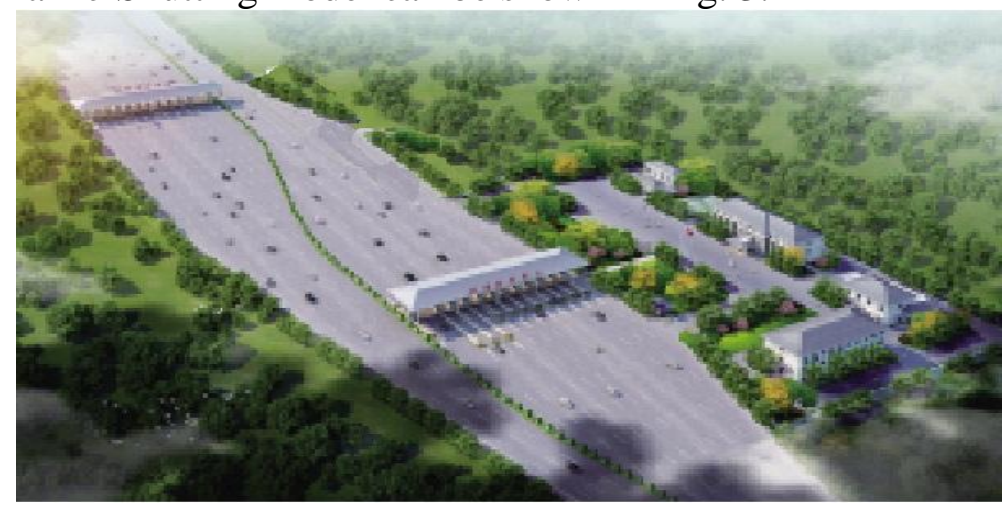

Fig. 3. diagram of Traffic-Shutting model

\subsection{Traffic Linear Model}

The traffic flow can be equivalent to a consequent fluid consists of a series of cars, which can be described by following formula:

$$
q=v k
$$

Through the statistical analysis, Greenshields put forward a linear model of the relation between $\mathrm{v}$ and $\mathrm{k}$ :

$$
v=v_{f}\left(1-k / k_{j}\right)
$$

We obtain the relation between $\mathrm{q}$ and $\mathrm{k}$ :

$$
q=v_{j} k\left(1-k / k_{j}\right)
$$

To demonstrate better the change in flow rate with traffic density, the graph is shown in Fig. 4. 


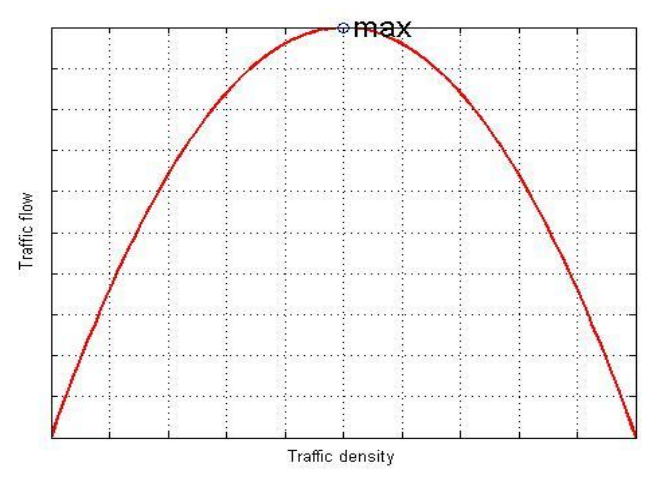

Fig. 4. The change in flow rate with traffic density

From the figure above, we can come to the conclusion that flow rate will reach the maximum value when $k=k_{c}=\frac{k_{j}}{2}$.

We can also get the relation between $\mathrm{q}$ and $\mathrm{v}$ :

$$
q=k_{j} v\left(1-\frac{v}{v_{f}}\right)
$$

As the point of our problem is the heavy traffic when vehicles merge after toll, we simplify the model by considering the flow rate mainly after the critical density. Therefore, the core idea of our model is to reduce the density by decrease the traffic density to $k_{c}$, for the purpose of growing the throughput, which is the theory of our Traffic-Shutting model.

The Calculation of Traffic Flow:

In this section, we test the Traffic-Shutting model based on the throughput of vehicles. We simplify our model as shown in Fig. 5.

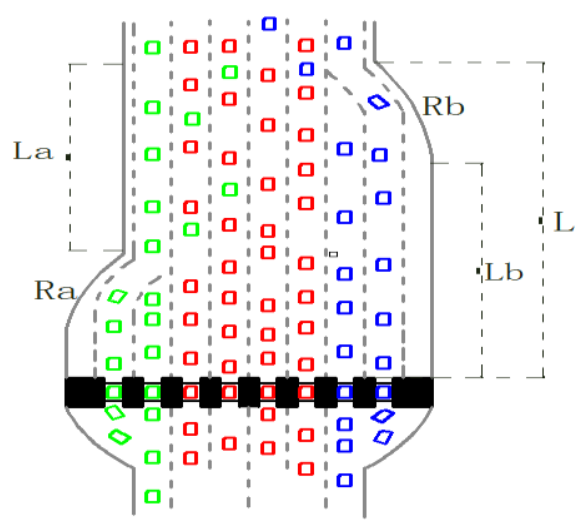

Fig. 5. The diagram drawn by AutoCAD

Based on Law of Acceleration, we can get following speed expression:

$$
v_{1}=\sqrt{v_{0}^{2}+2 a L_{a}} \quad v_{2}=\sqrt{v_{0}^{2}+2 a L} \quad v_{3}=\sqrt{v_{0}^{2}+2 a L_{b}}
$$

The corresponding time expression can be figured out:

$$
t_{1}=\frac{\pi R_{a}}{2 v_{0}}+\frac{v_{1}-v_{0}}{a} \quad t_{2}=\frac{v_{2}-v_{0}}{a} \quad t_{3}=\frac{v_{3}-v_{0}}{a}+\frac{\pi R_{b}}{2 v_{3}}
$$

Obviously, $t_{2}$ is the minimum among them:

Applying the staying time we get from Queuing Theory, the $\Delta t$ between each line can be figured out as following:

$$
T_{1}=\Delta t_{12}+w_{s}=t_{1}-t_{2}+w_{s}
$$




$$
T_{2}=\Delta t_{23}+w_{s}=t_{3}-t_{2}+w_{s}
$$

Here we use $q_{1}, q_{2}, q_{3}$ to represent the value of flow in $a_{1}, a_{2}, a_{3}$.

We transfer the calculation of total flow into the calculation of block area based on our definition of traffic flow block (Fig. 6).

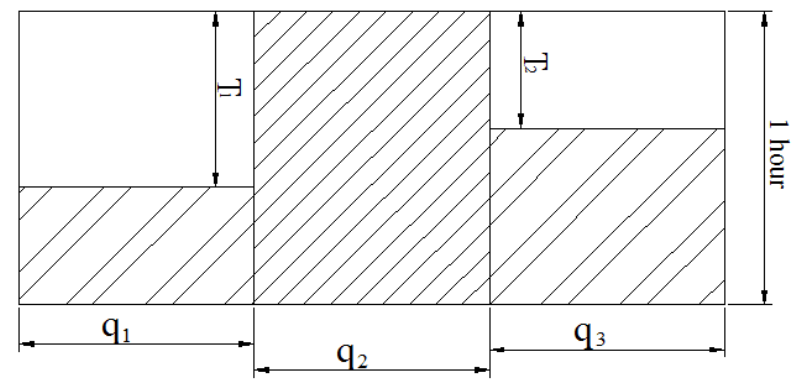

Fig. 6. The Diagram of the Calculation of Traffic Flow Block

The expression of total flow can be figured out as following:

$$
Q=\frac{1-T_{1}}{1} q_{1}+q_{2}+\frac{1-T_{2}}{1} q_{3}
$$

\section{Summary}

The time difference brought about by the shunting make it possible to decrease the density, so as to increase the throughput of vehicles.

\section{References}

[1] Jian Li, Li-li Liu, Bin Hu, Jian-cheng Weng, Jian Rong. (2012) Method for Determining Expressway Mixed Toll Station Traffic Capacity Based on VISSIM Simulation Model. Highway (NO.7) :184-187.

[2] Qi-yuan Jiang, Jin-xing Xie, Jun Ye. Mathematical Model. Higher Education Press: 38-39. 